\title{
Influencia de la personalidad y el estilo de aprendizaje en la elección de especialidad médica
}

\author{
Marcela Bitran $C^{a}$, Denisse Zúñiga $P^{b}$, \\ Monserrat Lafuente G, Paola Viviani G C , Beltrán Mena C. \\ Influence of personality and learning \\ styles in the choice of medical \\ specialty
}

Background: Several studies indicate that doctors who work in the same area of the medical profession tend to behave somehow similarly. Thus, it has been suggested that personality relates to the medical specialty choice. However, it is not known whether people selfselect into the medical specialties according to their personality or the professional practice in a particular field influences their behavior. Aim: To explore the possible association between the graduate's personality features and learning styles and their chosen specialty. Subjects and Methods: The psychological preferences and learning styles of 65 students of the 2001-graduating cohort of the Pontificia Universidad Católica de Chile School of Medicine were evaluated with the Myers Briggs Type Indicator and the Kolb Learning Style Inventory, respectively. These variables were correlated with the information of their specialty choice or occupation two years after graduation. Results: Graduates distributed unevenly in different areas of the medical profession. Surgical specialties concentrated a larger proportion of extraverted, intuitive and structured doctors, whereas in Pediatrics and Internal Medicine predominated intuitive and people-oriented MD's. Primary Care concentrated individuals with introverted, intuitive and flexible attitudes. Convergent learners (interested in problem-solving) preferred Surgery and Primary Care whereas Assimilator learners (abstract-reflexive) chose more frequently Internal Medicine, Pediatrics and Psychiatry. Conclusions: According to their personality and learning style, graduates tend to self-select into different medical specialties. This information may help medical graduates to guide their specialty choice process, and medical educators to develop learning experiences that take into account the individual differences of their residents (Rev Méd Chile 2005; 133: 1191-9).

(Key Words: Learning; Personality, Specialties, medical; Specialties, surgical)
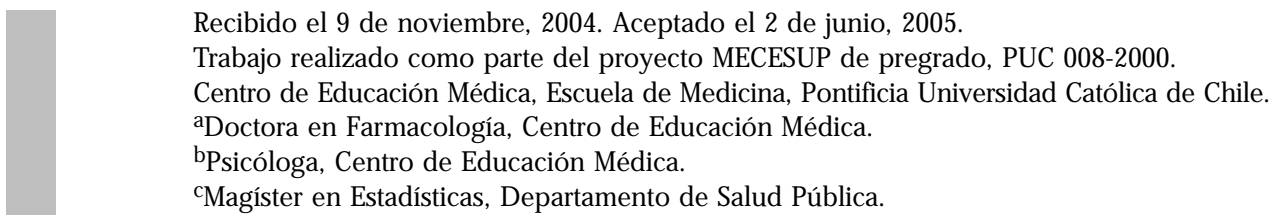

Correspondencia a: Marcela Bitran C. Centro de Educación Médica, Escuela de Medicina, Pontificia Universidad Católica de Chile. Edificio Escuela de Medicina. Alameda № 340, 6o piso, Santiago-Centro. Fono: 354-3811. Fax: 633-1457.

E-mail: mbitran@med.puc.cl 
La elección de especialidad médica ha sido motivo de estudio, particularmente en las escuelas de medicina norteamericanas que han indagado sistemáticamente en este campo desde la década 1960691-3. El hecho que en las distintas especialidades médicas se encuentren individuos con características de personalidad y estilos similares, ha llevado a algunos investigadores a proponer que estas variables inciden en la elección de especialidad ${ }^{3-}$ 19. El problema con estos estudios es que, por haberse realizado con especialistas con años de experiencia profesional, no es posible establecer si las características asociadas con una especialidad son pre-existentes a la elección, o derivan de la práctica de ésta. En otras palabras, no sabemos si los cirujanos se vuelven más 'prácticos y decididos' porque ejercen la cirugía, o la cirugía es una especialidad que atrae a los médicos 'prácticos y decididos'.

En nuestro país no existen estudios que evalúen la relación entre la personalidad y la elección de especialidad médica. Más aún, son escasas las publicaciones relativas a la elección de especialidad, y éstas se centran, ya sea en la distribución de especialistas en el país ${ }^{20,21}$ o en el perfil de los programas de especialización ${ }^{31}$.

Como parte de nuestro interés general respecto de la influencia de la personalidad y los estilos de aprendizaje en la formación de los estudiantes de medicina ${ }^{22}$, nos interesó determinar si las características de personalidad y estilos de aprendizaje influían también en la elección de especialidad médica. Para ello, determinamos el patrón de tipos psicológicos y estilos de aprendizaje de una cohorte de estudiantes (al egreso), y estudiamos la relación entre estas variables personales y el programa de especialización u ocupación en el que se encontraban dos años más tarde. En este manuscrito reportamos las asociaciones encontradas entre estas variables.

\section{MATERIAL Y MÉTODO}

Población. Participaron 65 de los 78 estudiantes de la cohorte de egreso 2001 de la Escuela de Medicina de la Pontificia Universidad Católica de Chile (25 $\pm 1,2$ años al egreso, $40 \%$ mujeres). Se incluyó a todos los estudiantes de 70 año que estuvieron presentes durante la aplicación de los tests y que consintieron en participar en este estudio. En dicha ocasión, se les solicitó que indicaran la especialidad o actividad que deseaban realizar una vez titulados. Dos años después de la titulación, se les contactó para conocer su ocupación.

Variables psicológicas. Las características de personalidad se evaluaron con el Inventario de Tipos Psicológicos de Myers y Briggs (MBTI forma G, versión en Español ${ }^{23-25}$, y los estilos de aprendizaje con el Inventario de Estilos de Aprendizaje de Kolb (LSI, versión en Español) ${ }^{26,27 .}$

El MBTI indica las preferencias psicológicas individuales y a partir de éstas se infiere el estilo cognitivo y tipo psicológico ${ }^{23-25}$. La preferencia es definida como la tendencia natural a actuar según uno $\mathrm{u}$ otro polo de 4 dimensiones psicológicas independientes: 1) la fuente de motivación (con sus polos Extraversion e Introversion), 2) la percepción de información (Sensing vs Intuition), 3) el discernimiento (Thinking vs Feeling) y 4) la actitud ante la vida (Judging vs Perceiving). Las dimensiones 1 y 4 se denominan orientaciones y las del medio, funciones cognitivas.

Respecto de las orientaciones, hay quienes se motivan mejor en el mundo exterior, en contacto con la gente (Extraversion, [E]) y otros, en el mundo interno y personal (Introversion, [I]). Análogamente, algunas personas viven de manera más estructurada y planificada (Judging, (J]) que otras, que prefieren mantener sus opciones abiertas (Perceiving, [P]). Respecto de las funciones cognitivas, algunas personas perciben la información prestando más atención a los detalles y aspectos concretos (Sensing, [S]) y otros, a los patrones generales y las posibilidades (Intuition, [N]). Por otra parte, al tomar una decisión, hay quienes privilegian los aspectos lógicos y objetivos (Thinking, [T]), o los personales y subjetivos (Feeling, [F]).

Los estilos cognitivos emergen de la combinación de las funciones cognitivas y son: 1) 'práctico -concreto' [ST]; 2) 'empático-concreto' [SF]; 3) lógico-ingenioso' [NT] y 4) 'empático-perceptivo' [NF].

Finalmente, el tipo psicológico se origina de la combinación de las 4 preferencias. Por ejemplo, el tipo INTJ caracteriza a una persona con preferencias Introversion, Intuition, Thinking y Judging. 
Dieciséis tipos psicológicos surgen de todas las combinaciones posibles.

Por otra parte, el LSI identifica cuatro tipos de aprendices según su modo de adquirir y utilizar la información, a saber: Asimilador, Convergente, Divergente y Acomodador ${ }^{26,27}$. El Asimilador (abstracto-reflexivo) tiende a aprender sistematizando la información en teorías unificadoras 0 patrones, y reflexiona acerca de ellos sin mucho interés en su aplicación práctica. El Convergente (abstracto-activo) aprende al aplicar el conocimiento a problemas, luego de generar modelos hipotéticos. El Divergente (concreto-reflexivo) tiene facilidad para aprender de la experiencia una vez que la ha considerado desde múltiples perspectivas y el Acomodador (concreto-activo) aprende mejor haciendo.

Tanto el MBTI como el LSI son instrumentos metodológicamente probados que se han utiliza- do ampliamente en investigación en educación médica en los Estados Unidos ${ }^{1,2,8,9}$ y en nuestra propia escuela desde el año $2000^{22,28,29}$.

Estadística. Se utilizaron las pruebas estadísticas ' $t$ ', Chi cuadrado y Fisher, según correspondiera.

\section{RESULTADOS}

Ocupación. Dos años después de titularse, los médicos que egresaron el año 2001 se encuentran dedicados a diversas ocupaciones (Tabla 1). Veintitrés por ciento se desempeña como Médico General de Zona y 77\% se encuentra realizando un programa de especialización. En general, la especialidad más frecuentemente elegida es Medicina Interna (MI), seguida por Pediatría (Pd), Cirugía (Cx) y Psiquiatría (Ps) (Tabla 2). En

Tabla 1. D istribución de los médicos según su ocupación a dos años de titularse

\begin{tabular}{|c|c|c|c|c|}
\hline \multirow[b]{2}{*}{ Ocupación } & \multirow[b]{2}{*}{ Código } & \multirow[b]{2}{*}{$\mathrm{n}$} & \multicolumn{2}{|c|}{$\%$ médicos } \\
\hline & & & x ocup & x área \\
\hline Médico General de Zona & MGZ & 15 & 23 & 23 \\
\hline \multicolumn{5}{|l|}{ Programa de Especialización } \\
\hline Medicina Interna & MI & 7 & 11 & \\
\hline Neurología & MI & 2 & 3 & \\
\hline Radiología & MI & 2 & 3 & \\
\hline Medicina Familiar Adulto & MI & 1 & 2 & 19 \\
\hline Pediatría & $\mathrm{Pd}$ & 7 & 11 & \\
\hline Medicina Familiar Niño & $\mathrm{Pd}$ & 2 & 3 & \\
\hline Neurología Infantil & $\mathrm{Pd}$ & 1 & 2 & 16 \\
\hline Cirugía & $\mathrm{Cx}$ & 3 & 5 & \\
\hline Traumatología y Ortopedia & $\mathrm{Cx}$ & 3 & 5 & \\
\hline Anestesiología & $\mathrm{Cx}$ & 2 & 3 & \\
\hline Neurocirugía & $\mathrm{Cx}$ & 1 & 2 & 15 \\
\hline Psiquiatría & Ps & 5 & 8 & 8 \\
\hline Dermatología & Otras & 2 & 3 & \\
\hline Investigación Básica & Otras & 2 & 3 & \\
\hline Obstetricia y Ginecología & Otras & 2 & 3 & \\
\hline Oftalmología & Otras & 2 & 3 & \\
\hline Otorrinolaringología & Otras & 2 & 3 & \\
\hline Nutrición & Otras & 1 & 2 & \\
\hline Laboratorio Clínico & Otras & 1 & 2 & \\
\hline \multirow[t]{2}{*}{ Administración en Salud } & Otras & 2 & 3 & 22 \\
\hline & & 65 & 100 & 100 \\
\hline
\end{tabular}

MGZ: Médico General de Zona, MI: Medicina Interna, Pd: Pediatría, Cx: Cirugía, y Ps: Psiquiatría. 
Tabla 2. D istribución de los médicos según su sexo y ocupación

\begin{tabular}{|lccc|}
\hline & $\begin{array}{c}\text { Hombres } \\
(\mathrm{n}=39)\end{array}$ & $\begin{array}{c}\text { Mujeres } \\
(\mathrm{n}=26)\end{array}$ & $\begin{array}{c}\text { Todos } \\
(\mathrm{n}=65)\end{array}$ \\
\hline MGZ & 28 & 15 & 23 \\
MI & 18 & 19 & 19 \\
Pd & 8 & 27 & 16 \\
Cx & 15 & 12 & 15 \\
Ps & 10 & 4 & 8 \\
Otras & 21 & 23 & 22 \\
& 100 & 100 & 100 \\
\hline
\end{tabular}

MGZ: Médico General de Zona, MI: Medicina Interna, Pd: Pediatría, Cx: Cirugía, y Ps: Psiquiatría.

Tabla 3. D istribución de los médicos según sus preferencias y ocupación

\begin{tabular}{|c|c|c|c|c|c|c|}
\hline \multirow{2}{*}{$\begin{array}{l}\text { Preferencias } \\
\text { Psicológicas }\end{array}$} & \multicolumn{6}{|c|}{ Programa Especialización } \\
\hline & $\begin{array}{l}\text { TODAS } \\
(\mathrm{n}=65)\end{array}$ & $\begin{array}{c}\text { MGZ } \\
(n=15)\end{array}$ & $\begin{array}{c}\text { MI } \\
(n=12)\end{array}$ & $\begin{array}{c}\mathrm{Pd} \\
(\mathrm{n}=10)\end{array}$ & $\begin{array}{c}\mathrm{Cx} \\
(\mathrm{n}=9)\end{array}$ & $\begin{array}{c}\text { Ps } \\
(n=5)\end{array}$ \\
\hline $\begin{array}{l}\text { Extraversion } \\
\text { Introversion }\end{array}$ & $\begin{array}{l}52 \\
48\end{array}$ & $\begin{array}{l}23 \\
73 \text { a }\end{array}$ & $\begin{array}{l}50 \\
50\end{array}$ & $\begin{array}{l}60 \\
40\end{array}$ & $\begin{array}{l}78 \\
22\end{array}$ & $\begin{array}{l}40 \\
60\end{array}$ \\
\hline $\begin{array}{l}\text { Sensing } \\
\text { Intuition }\end{array}$ & $\begin{array}{l}50 \\
50\end{array}$ & $\begin{array}{ll}13 & \\
87 & a, b\end{array}$ & $\begin{array}{l}33 \\
67 \quad \mathrm{a}\end{array}$ & $\begin{array}{l}70 \\
30\end{array}$ & $\begin{array}{r}100 \\
0\end{array}$ & $\begin{array}{l}60 \\
40\end{array}$ \\
\hline $\begin{array}{l}\text { Thinking } \\
\text { Feeling }\end{array}$ & $\begin{array}{l}77 \\
23\end{array}$ & $\begin{array}{l}87 \\
13\end{array}$ & $\begin{array}{l}58 \\
42 \quad \mathrm{a}\end{array}$ & $\begin{array}{l}60 \\
40\end{array}$ & $\begin{array}{r}100 \\
0\end{array}$ & $\begin{array}{r}100 \\
0\end{array}$ \\
\hline $\begin{array}{l}\text { Judging } \\
\text { Perceiving }\end{array}$ & $\begin{array}{l}67 \\
33\end{array}$ & $\begin{array}{l}33 \\
67 \quad a, b\end{array}$ & $\begin{array}{l}67 \\
33\end{array}$ & $\begin{array}{l}80 \\
20\end{array}$ & $\begin{array}{l}89 \\
11\end{array}$ & $\begin{array}{l}80 \\
20\end{array}$ \\
\hline
\end{tabular}

MGZ: Médico General de Zona, MI: Medicina Interna, Pd: Pediatría, Cx: Cirugía, y Ps: Psiquiatría. ${ }^{\text {Respecto de }}$ $\mathrm{Cx}$ y ${ }^{b}$ respecto de Pd. Las diferencias corresponden a valores de $\mathrm{p}$ entre 0,0001 y 0,04 .

Tabla 4. D istribución de los médicos según sus estilos cognitivos y ocupación

\begin{tabular}{|c|c|c|c|c|c|c|}
\hline \multirow{3}{*}{$\begin{array}{l}\text { Estilos } \\
\text { Cognitivos }\end{array}$} & \multirow[b]{3}{*}{$\begin{array}{l}\text { TODAS } \\
(n=65)\end{array}$} & \multirow[b]{3}{*}{$\begin{array}{c}\text { MGZ }^{a, b} \\
(n=15)\end{array}$} & \multirow{2}{*}{\multicolumn{2}{|c|}{$\%$ Procrama }} & \multirow[b]{2}{*}{ alización } & \multirow[b]{3}{*}{$\begin{array}{c}\text { Ps } \\
(n=5)\end{array}$} \\
\hline & & & & & & \\
\hline & & & $\begin{array}{l}\mathrm{MI}^{\mathrm{a}, \mathrm{b}} \\
(\mathrm{n}=12)\end{array}$ & $\begin{array}{c}\mathrm{Pd}^{\mathrm{a}} \\
(\mathrm{n}=10)\end{array}$ & $\begin{array}{c}\mathrm{Cx} \\
(\mathrm{n}=9)\end{array}$ & \\
\hline ST (práctico-concreto) & 40 & 13 & 33 & 30 & 100 & 60 \\
\hline NT (lógico-ingenioso) & 38 & 74 & 24 & 30 & - & 40 \\
\hline SF (empático-concreto) & 12 & - & - & 40 & - & - \\
\hline \multirow[t]{2}{*}{ NF (empático-perceptivo) } & ) 10 & 13 & 43 & - & - & - \\
\hline & 100 & 100 & 100 & 100 & 100 & 100 \\
\hline
\end{tabular}

S: Sensing, N: Intuition, T: Thinking y F: Feeling. MGZ: Médico General de Zona, MI: Medicina Interna, Pd: Pediatría, Cx: Cirugía, y Ps: Psiquiatría. ${ }^{a}$ Respecto de $\mathrm{Cx}$ y ${ }^{\mathrm{b}}$ respecto de Pd. Las diferencias corresponden a valores de $\mathrm{p}$ entre 0,0001 y 0,04 . 
conjunto, las 3 especialidades básicas (MI, Pd y Cx) concentran a $50 \%$ de los médicos (Tabla 1 ).

Al estratificar por sexo, observamos que hay mayor proporción de hombres en los Médicos Generales de Zona (28\% vs 15\%, Tabla 2). En los programas de especialización, las diferencias más notables se dan en Pediatría, escogida preferentemente por mujeres ( $27 \%$ vs $8 \%)$, y Psiquiatría, por los hombres (10\% vs $4 \%$ ) (Tabla 2$)$.

A dos años de titularse, $77 \%$ de los médicos se encuentra realizando la actividad a la que -según indicó al momento del egreso- deseaba dedicarse (datos no mostrados) ${ }^{*}$. Esta proporción aumenta a $92 \%$ si se incluyen solamente los médicos que se encuentran en un programa de especialización.

\section{Preferencias psicológicas}

a) Distribución. En la población total, igual porcentaje de médicos tiene preferencia por Extraversion que por Introversion (Tabla 3). Lo mismo ocurre con la percepción de la información (S vs $\mathrm{N})$. En cambio, respecto al discernimiento (T-F) y la actitud frente a la vida (J-P), la distribución es desigual: la mayoría prefiere T y J (Tabla 3).

b) Relación con la elección de especialidad. Los médicos se distribuyen de manera desigual en la distintas especialidades o actividades en lo que respecta a su modo de percibir la información (Tabla 3). Los 'médicos generales' son significativamente más intuitivos (N) que los 'cirujanos' y 'pediatras'. De hecho, en Cirugía, no hay médicos con percepción intuitiva. Los 'internistas' también son distintos a los 'cirujanos' en cuanto tienen una mayor proporción de intuitivos.

Respecto al modo de discernir, también existen diferencias: en Cirugía y Psiquiatría no existen médicos con discernimiento Feeling $(\mathrm{F})$, los que constituyen más de $40 \%$ de los 'médicos internistas' y 'pediatras' (Tabla 3).

Respecto de sus orientaciones, los 'médicos generales' se diferencian de los 'cirujanos' por ser más introvertidos, y de los 'cirujanos' y los 'pediatras' por ser más flexibles (Tabla 3).

\section{Estilos cognitivos}

a) Distribución y relación con la elección de especialidad. En todas las ocupaciones, predomi- nan los individuos con estilos cognitivos racionales (ST y NT). Sin embargo, la proporción de estos estilos varía según la ocupación (Tabla 4). El caso más destacable es el de Cirugía: 100\% de los médicos es 'lógico-concreto' (ST). En contraste, la mayoría de los 'médicos generales' tienen estilo 'lógico-ingenioso' (NT).

Medicina Interna y Pediatría son los únicos programas de especialización en que hay médicos con estilos empáticos. En los 'internistas' predominan los 'empático-perceptivos' (NF), mientras que en los 'pediatras', los 'empático-concretos' (SF).

\section{Tipos psicológicos}

a) Distribución. En la población general están representados 15 de los 16 tipos psicológicos (Tabla 5). En tres de éstos (ISTJ, INTP y ESTJ) se concentra más de la mitad de los médicos. El tipo más frecuente es el ESTJ (21\%), característico de personas extravertidas, prácticas, lógicas y estructuradas. Le sigue el INTP (17\%), que corresponde al perfil de personas reservadas, intuitivas, objetivas y flexibles. En tercer lugar (15\%), está el tipo ISTJ: introvertido, sensorial, lógico y estructurado (Tabla 5).

Hay que agregar también que en la población general hay una proporción cercana a $10 \%$ de los tipos INTJ y ESFJ. El INTJ corresponde al perfil de personas introvertidas, intuitivas, lógicas y estructuradas; mientras que el ESFJ es característico de personas extravertidas, prácticas, empáticas y estructurados. En conjunto, los 5 tipos psicológicos descritos (ISTJ, INTJ, ESTJ, INTJ y ESFJ) representan a 70\% de la población. El factor común de ellos es la marcada tendencia a hacer juicios lógicos y objetivos (T), y a vivir de manera estructurada (J). Estas dos características no son propias del ISFP, único tipo que no está presente en nuestros egresados, y que corresponde a personas reservadas, prácticas, empáticas y flexibles.

b) Relación con la elección de especialidad. La diversidad y distribución de los tipos psicológicos cambia según la especialización u ocupación elegida. El tipo más frecuente de los 'médicos generales' es el INTP, el de los 'pediatras' el ESFJ y el de 'cirujanos' y 'psiquiatras', el ESTJ. Medicina Interna se diferencia de los demás programas por tener médicos con una mayor diversidad de tipos

*Esto fue consignado al momento de administrar los tests. 
psicológicos y sin claro predominio de uno en particular (Tabla 5).

Estilos de aprendizaje

a) Distribución y relación con la elección de especialidad. De los 4 estilos de aprendizaje, los más comunes son el Asimilador y el Convergente (79\%, Tabla 6). Estos dos estilos predominan también en cada una de las ocupaciones estudiadas, sin embargo, la proporción relativa de Asimiladores y Convergentes varía: en los 'médicos generales' y 'cirujanos' la relación es de 1:2 y 1:3,

Tabla 5. D istribución de los médicos según sus tipos psicológicos y ocupación

\begin{tabular}{|c|c|c|c|c|c|c|}
\hline \multirow[b]{3}{*}{ Tipos } & \multirow[b]{3}{*}{$\begin{array}{l}\text { TODAS } \\
(\mathrm{n}=65)\end{array}$} & \multirow[b]{3}{*}{$\begin{array}{c}\text { MGZ } \\
(n=15)\end{array}$} & \multicolumn{3}{|c|}{$\%$} & \multirow[b]{3}{*}{$\begin{array}{c}\text { Ps } \\
(n=5)\end{array}$} \\
\hline & & & \multicolumn{3}{|c|}{ Programa Especialización } & \\
\hline & & & $\begin{array}{c}\mathrm{MI} \\
(\mathrm{n}=12)\end{array}$ & $\begin{array}{c}\mathrm{Pd} \\
(\mathrm{n}=10)\end{array}$ & $\begin{array}{c}\mathrm{Cx} \\
(\mathrm{n}=9)\end{array}$ & \\
\hline ISTJ & 15 & 13 & 17 & 10 & 22 & 20 \\
\hline ISFJ & 2 & & & & 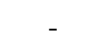 & - \\
\hline INFJ & 3 & 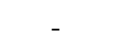 & 17 & - & - & - \\
\hline INTJ & 9 & 13 & 8 & 10 & - & 20 \\
\hline ISTP & 2 & - & 8 & - & - & - \\
\hline ISFP & - & - & - & - & - & - \\
\hline INFP & 2 & 7 & - & & - & - \\
\hline INTP & 17 & 40 & - & 20 & - & 20 \\
\hline ESTP & 2 & - & - & - & 11 & - \\
\hline ESFP & 2 & - & - & - & - & - \\
\hline ENFP & 5 & 7 & 17 & - & - & - \\
\hline ENTP & 6 & 13 & 8 & & & - \\
\hline ESTJ & 21 & - & 8 & 20 & 67 & 40 \\
\hline ESFJ & 8 & - & 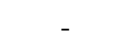 & 40 & - & - \\
\hline ENFJ & 3 & - & 8 & - & - & - \\
\hline ENTJ & 6 & 7 & 8 & - & - & - \\
\hline & 100 & 100 & 100 & 100 & 100 & 100 \\
\hline
\end{tabular}

MGZ: Médico General de Zona, MI: Medicina Interna, Pd: Pediatría, Cx: Cirugía, y Ps: Psiquiatría. E: Extraversion, I: Introversion, S: Sensing, N: Intuition, T: Thinking, F: Feeling, J: Judging y P: Perceiving. Nota: el tipo psicológico se origina de la combinación de 4 preferencias psicológicas.

Tabla 6. D istribución de los médicos según sus estilos de aprendizaje y ocupación

\begin{tabular}{|lcccccc|}
\hline \multirow{2}{*}{$\begin{array}{l}\text { Estilos } \\
\text { Aprendizaje }\end{array}$} & \multicolumn{5}{c}{$\begin{array}{c}\% \\
\text { Programa Especialización }\end{array}$} \\
\cline { 4 - 7 } & $\begin{array}{c}\text { TODAS } \\
(\mathrm{n}=62)\end{array}$ & $\begin{array}{c}\text { MGZ } \\
(\mathrm{n}=14)\end{array}$ & $\begin{array}{c}\mathrm{MI} \\
(\mathrm{n}=11)\end{array}$ & $\begin{array}{c}\text { Pd } \\
(\mathrm{n}=10)\end{array}$ & $\begin{array}{c}\mathrm{Cx} \\
(\mathrm{n}=8)\end{array}$ & $\begin{array}{c}\text { Ps } \\
(\mathrm{n}=5)\end{array}$ \\
\hline Asimilador & 44 & 29 & 45 & 60 & 25 & 60 \\
Convergente & 35 & 57 & 18 & 30 & 75 & 20 \\
Divergente & 11 & - & 18 & 10 & - & - \\
Acomodador & 10 & 14 & 18 & - & - & 20 \\
& 100 & 100 & 100 & 100 & 100 & 100 \\
\hline
\end{tabular}

MGZ: Médico General de Zona, MI: Medicina Interna, Pd: Pediatría, Cx: Cirugía, y Ps: Psiquiatría. 
respectivamente. En cambio, en Medicina Interna, Pediatría y Psiquiatría la relación Asimilador: Convergente se invierte, siendo 2,4:1 en MI, 2:1 en Pd y 3:1 en Ps.

Aunque el análisis estadístico de la distribución de los 4 estilos de aprendizaje según la ocupación no arrojó diferencias significativas (probablemente debido al reducido tamaño muestral), es aparente que en ninguno de los estilos, la frecuencia observada corresponde a una distribución al azar.

\section{DisCUSIÓN}

La distribución de los médicos egresados de la Universidad Católica el año 2001 en las 4 especialidades médicas básicas sigue, en términos generales, un patrón similar a la distribución de cupos ofrecidos anualmente por las distintas instituciones acreditadas del país $(\mathrm{M}>\mathrm{Pe} \approx \mathrm{CX}>\mathrm{OG})^{30}$. Sin embargo, en nuestra población, la proporción 'intemistas':' obstetras' es mayor que la oferta relativa de becas para dichas especialidades $\left(6: 1 \mathrm{v} / \mathrm{s} 3,6: 1^{30}\right)$, hecho que puede interpretarse como un menor interés relativo de nuestros egresados por obstetricia y ginecología (OG).

Analizada esta información en su conjunto, parece razonable concluir que el mercado ejerce una influencia en la elección de especialidad médica de nuestros egresados. Hay que considerar además, que el interés por los distintos programas de especialización está cambiando como resultado de la reforma de salud, las dificultades de financiamiento y el aumento de problemas legales relacionados con el ejercicio de la profesión. Es así como parece haber un interés creciente por especialidades derivadas, en desmedro de las básicas como OG $^{30,31}$.

Habida cuenta de la importancia de las variables antes analizadas, los resultados de este estudio indican que las características de personalidad también influyen en la elección de especialidad médica. En Medicina General, por ejemplo encontramos más médicos introvertidos, intuitivos y flexibles que en Cirugía; especialidad elegida principalmente por personas extravertidas, sensoriales y estructuradas. Por otra parte, los médicos más interesados en los aspectos humanos y subjetivos, optan preferentemente por Pediatría y Medicina Interna.
Estas diferencias se manifiestan también en los estilos cognitivos. Todos los becados de Cirugía son 'lógico-concretos', mientras que la mayoría de los 'médicos generales' son 'lógico-ingeniosos'. Por su parte los 'psiquiatras' se distribuyen entre estos dos estilos, que tienen en común el discernimiento lógico. Por el contrario, Pediatría y Medicina Interna se distinguen por contar con becados 'empáticos': $40 \%$ de los 'pediatras' son 'empático-concretos' y 40\% de los 'internistas', 'empático-perceptivos'.

A pesar de que en los estilos de aprendizaje no se observaron diferencias significativas, la tendencia es que los médicos Convergentes (que aprenden mejor resolviendo problemas) prefieren la Medicina General y la Cirugía, mientras, los Asimiladores (aprendices más bien teóricos y reflexivos) optan por la Medicina Interna, la Pediatría y la Psiquiatría; lo observado es sólo una tendencia sin significancia estadística.

Gran parte de las asociaciones encontradas en este estudio parecen tener sentido al considerar las demandas características de las distintas ocupaciones. Por ejemplo, en Cirugía se requieren médicos capaces de lidiar en forma asertiva, eficiente y oportuna con problemas concretos e imprevistos. En este escenario, es esperable que se desempeñen mejor las personas pragmáticas y resolutivas, para quienes los problemas son un desafío. Por otra parte, Medicina Interna y Pediatría requieren habilidades diferentes: la facilidad para generar una relación de confianza con el paciente y para considerarlo como ser integral y complejo, desde múltiples puntos de vista. Estas son las habilidades naturales de los individuos con preferencia Feeling, cuyo interés primordial es mantener relaciones cercanas y armoniosas.

Menos evidente es la correspondencia entre las características de los 'médicos generales' y los requerimientos propios de su desempeño. Esto deriva del hecho que, por definición, las demandas a las que se ve expuesto un 'médico general' son mucho más variables que las de un especialista. Sin embargo, parece razonable pensar que el ingenio, la capacidad de resolver los problemas lógicamente, y la actitud abierta y flexible, son ventajas adaptativas para un médico que tendrá que trabajar en localidades aisladas y con recursos limitados.

Afirmar que las características aquí estudiadas se relacionan con la elección de ocupación y de 
especialidad, requiere demostrar que -en efectonuestros egresados han elegido libremente su ocupación o especialidad. Esto es así para la mayoría de los becados: 92\% de ellos está en el programa que deseaba seguir, según indicó al momento de egresar. La situación de los 'médicos generales' es distinta: sólo la mitad de ellos eligió ser 'médico general' y el resto, o no sabe a lo que quiere dedicarse, o está en una suerte de compás de espera hasta conseguir una beca en el programa de su interés.

Considerando la dura competencia que existe en nuestro país por las becas de especialización, la coincidencia encontrada en este estudio entre el programa elegido y el obtenido, nos parece excepcional y suficientemente alta para afirmar que la ocupación (al menos en el caso de los becados) ha sido elegida libremente. Aun cuando el supuesto de libre elección no se aplique a todos los 'médicos generales', parece razonable que la perspectiva de ejercer en regiones aisladas $\mathrm{y}$ en condiciones a menudo inciertas, sea un escenario profesional más concebible para quienes, como nuestros egresados, están más abiertos al cambio y le es más fácil ver posibilidades donde otros sólo verían dificultades.

Estudios realizados en más de 3.000 médicos norteamericanos, son coincidentes con el nuestro al indicar que el modo de percibir la información y de discernir están relacionadas con la especiali$\operatorname{dad}^{1,4,5,25}$. En los 'pediatras' y en los 'cirujanos' norteamericanos hay una sobre-representación de individuos con percepción sensorial, mientras que en los 'internistas' predominan los individuos con percepción intuitiva ${ }^{1,4}$. Por otra parte, en las especialidades onientadas a la atención y cuidado del paciente (Medicina Familiar y Pediatría), es más probable encontrar médicos con discemimiento Feeling que en Cirugía, Psiquiatría y Medicina General ${ }^{1,4,5}$. En contraste con nuestros resultados,

\section{REFERENCIAS}

1. Stilwell NA, Waшick MM, Thal SE, Burleson JA. Myers-Briggs type and medical specialty choice: a new look at an old question. Teach Learn Med 2000; 121: 14-20.

2. Zeldow P, Daugherty S. Personality profiles and specialty choices of students from two medical los estudios norteamericanos muestran que los Psiquiatras son predominantemente intuitivos ${ }^{1,4,5}$.

Estudios de especialidad médica y estilos de aprendizaje realizados con profesionales norteamericanos coinciden con la tendencia evidenciada en este estudio, cual es que la mayoría de los cirujanos tiene un estilo de aprendizaje activo (Convergente o Acomodador), mientras que en los Psiquiatras predominan los estilos reflexivos (Asimilador o Divergente) ${ }^{8,9}$.

Es importante destacar que, a diferencia de los estudios citados que se realizaron con especialistas con más de 5 años de experiencia, nuestro estudio se realizó con médicos recién egresados, antes que iniciaran sus becas o actividad profesional. En consecuencia, los patrones de personalidad reportados en este manuscrito no son atribuibles a determinada práctica médica, sino al interés por especializarse en una u otra área de la clínica médica.

Tenemos clara conciencia que para establecer la generalizabilidad de nuestros resultados se requiere estudiar otras cohortes de egresados e, idealmente, realizar un estudio multicéntrico. Sin embargo, el hecho que los patrones aquí encontrados coincidan con los estudios internacionales da fuerza a la idea que estos resultados no son propios de esta cohorte en particular.

Conocer las asociaciones entre personalidad, estilos de aprendizaje, y la elección de especialidad, provee valiosa información tanto para los futuros profesionales como para los educadores médicos $\mathrm{y}$, particularmente, para los docentes encargados de los programas de especialización. Es nuestra opinión que esta información no debe ser utilizada con fines de admisión, sino para desarrollar una mirada que considere las diferencias individuales, de modo de proporcionar oportunidades de aprendizaje apropiadas para todos.

school classes. Acad Med 1991; 66: 283-7.

3. Coffin S, Bаввотт D. Early and final preferences for pediatrics as a specialty: A study of U.S. medical school graduates in 1983. Acad Med 1989; 64: 600-5.

4. WawCK MM, Cambre KM, RandaL HM. Personality type and medical specialty choice. J La State Med Soc 1999; 151: 463-9. 
5. Friedman C, Siatt L. New results relating the Myers-Briggs type indicator and medical specialty choice. J Med Educ 1988; 63: 325-7.

6. Lester W, WoLoschuk W, Juschia B, Mandin H. Assessing the psychological types of specialists to assist students in career choice. Acad Med 1995; 70: 932-3.

7. McGrath E, Zimet C. Female and male medical students differences in specialty choice selection and personality. J Med Educ 1977; 52: 293-300.

8. Plovnick M. Primary care career choices and medical student learning styles. J Med Educ 1975; 50: 849-55.

9. BaKER J, Reines H, WaLACE C. Learning style analysis in surgical training. Am Surg 1985; 51: 494-6.

10. Hojat M, Nasca T, Magee M, Feeney K, Pascual R, URBANO $F$ ET aL. A comparison of the personality profiles of internal medicine residents, physician role models, and the general population. Acad Med 1999; 74: 1327-33.

11. Krol D, Morris V, BetZ J, Cadman E. Factors influencing the career choices of physicians trained at Yale-New Haven Hospital from 1929 through 1994. Acad Med 1998; 73: 313-7.

12. Du M, Heymans R, Noordendoos G. Gender factors in the selection of training for a medical specialty. Ned Tijdschr Genneeskd 2000; 144: 129-33.

13. Wendel T, Godelas C, Prinz R. Are there differences in choosing a surgical career? Surgery 2003; 134: 591-6.

14. Campos-Outcalt, Senf J. Characteristics of medical schools related to the choice of family medicine as a specialty. Acad Med 1989; 64: 610-5.

15. Lieu T, Schroeder S, Altman D. Specialty choices at one medical school: Recent trends and analysis of predictive factors. Acad Med 1989; 64: 622-9.

16. Biand C, Meurer L, Maidonado G. Determinants of primary care specialty choice: a non-statistical metaanalysis of the literature. Acad Med 1995; 70: 620-41.

17. ReED V, JeRNSTEDT G, REBER E. Understanding and improving medical student specialty choice: a synthesis of the literature using decision theory as a referent. Teach Learn Med 2001; 13: 117-29.

18. Reich D, Uysal S, Bodian C, Gabriele S, Hibbard M, Gordon W ET AL. The relationship of cognitive, personality and academic measures to anesthesiology resident clinical performance. Anesth Analg 1999; 88: 1092-100.
19. Ward A, Kamien M, López D. Medical career choice and practice location: early factors predicting course completion, career choice and practice location. Med Educ 2004; 38: 239-48.

20. Román O. Un análisis de la situación actual de las especialidades médicas en Chile. Rev Méd Chile 2002; 130: 809-15.

21. MEDINA E. Especialidades médicas y especialistas en Chile. Rev Méd Chile 1990; 118: 1276-83.

22. Bitran M, Lafuente M, Zúñiga D, Viviani P, Mena B. ¿Influyen las características psicológicas y estilos de aprendizaje en el rendimiento académico de los estudiantes de Medicina? Un estudio retrospectivo. Rev Méd Chile 2004; 132: 1127-36.

23. Briggs I. MBTI: Inventario Tipológico Forma G. Manual. Madrid: TEA Ediciones. 1991.

24. Myers IB, MacCauley M, Quenk N, Hammer A. MBTI Manual: A guide to the development and use of de Myers-Briggs Type Indicator. Palo Alto, California: Consulting Psychologist Press, Inc. 1998.

25. Myers I, Myers P. Gifts Differing. Understanding Personality Type. Palo Alto: Davies-Black Publishing, 1980.

26. KоцB D. Inventario de los estilos de aprendizaje: Inventario autoevaluativo y su interpretación. Boston: Training Resources Group, Hay/Mc Ber, 1981.

27. Kоцв D. Experiential Learning: Experience as the Source of Learning and Development. New Jersey: Prentice Hall, PTR. 1984.

28. Bitran M, Zúñiga D, Lafuente M, Viviani P, Mena B. Tipos Psicológicos y Estilos de Aprendizaje de los estudiantes que ingresan a la Pontificia Universidad Católica de Chile. Rev Méd Chile 2003; 131: 1067-78.

29. Bitran M, Zúñiga D, Lafuente M, Viviani P, Mena B. Características psicológicas y estilos cognitivos de estudiantes de Medicina y de otras carreras de la Pontificia Universidad Católica de Chile. Rev Méd Chile 2004; 132: 809-15.

30. ASOFAMECH. Cuadro № 8. Documento interno de la Comisión de Acreditación de Centros y Programas de Postítulos, 2004.

31. Cuelo M, Oyarzún E, WidD R. Perfil actual y requisitos para un programa de especialización en Obstetricia y Ginecología en el nuevo milenio en Chile. Rev Chil Obstet Ginecol 2004; 69: 136-48. 